\title{
Advanced Models for Prediction of High Altitude Aero- Thermal Loads of a Space Re-entry Vehicle
}

\author{
R. Votta ${ }^{\mathrm{a}}$, A. Schettino ${ }^{\mathrm{a}}$ and A. Bonfiglioli ${ }^{\mathrm{b}}$ \\ ${ }^{a}$ CIRA Italian Aerospace Research Centre, Via Maiorise 81043 Capua (CE), Italy \\ ${ }^{b}$ Department of Environmental Engineering and Physics (DIFA), via dell'Ateneo Lucano, Potenza, Italy
}

\begin{abstract}
The analysis of the rarefaction effects in predicting the main aero-thermal loads of a Space re-entry vehicle is presented. It is well known that the Navier-Stokes equations fail in rarefied regimes and other approaches must be used. In the present paper different configurations have been simulated by using the Direct Simulation Monte Carlo method. Moreover, slip flow boundary conditions have been implemented in a Navier-Stokes code in order to extend the validity of the continuum approach to the transitional flow regime. Finally, bridging formulas for high altitude aerodynamics of winged bodies have been used. Firstly, two simple geometries have been analysed, specifically designed to study the phenomenon of shock wave boundary layer interaction: a hollow cylinder flare, for which some experiments are available; and a blunt-nosed flat plate/flap model designed and tested at the Italian Aerospace Research Centre. The other configurations taken into account are, respectively, an experimental winged re-entry vehicle and a capsule, for which global aerodynamic coefficients and local wall heating have been determined with different approaches. The NavierStokes code with slip flow boundary conditions has shown good predicting capabilities compared with experiments in the hollow cylinder flare case; however, for the winged vehicle and capsule cases, the CFD results are not fully satisfactory and the Monte Carlo method remains the most reliable approach, together with the bridging formula, that provides good results for the aerodynamic coefficients.
\end{abstract}

Keywords: Aerothermodynamics, Slip Flow, Heat Flux, DSMC, CFD.

PACS: 47.45.-n, 47.40.Ki, 47.45.Gx, 47.45.Gx

\section{INTRODUCTION}

The correct prediction of the aerodynamic and aerothermodynamic loads of a space vehicle at high altitude is an important feature to be taken into account in the design phase; the goal of the present paper is to summarize some relevant experiences made by the authors [1,2 and 3] in the analysis of different configurations in transitional and rarefied regime and to draw some conclusions useful for future activities.

The first vehicle configuration that has been considered is an experimental Unmanned Space Vehicle (USV) that is being designed at the Italian Aerospace Research Centre (CIRA) as a test bed for re-entry technologies. The hypersonic configuration of this eXperimental Flying Test Bed (FTB), named FTB-X, requires an high aerodynamic efficiency, necessary for realizing a trajectory significantly longer than the one of Space Shuttle, in order to obtain a large down-range. Then, a correct prediction of the aerothermodynamic parameters at high altitude is fundamental. A first version of the vehicle, namely FTB-1, was launched on February 2007 and April 2010 by means of a stratospheric balloon, and performed two flight experiments in transonic and low supersonic regime. FIGURE 1 shows four views of the FTB-X configuration named 3.9.2-FW-50, as it was defined at the end of the preliminary design phase [4].

The second configuration taken into account is the NASA ORION CEV capsule [5], see FIGURE 2, analysed in the frame of the RTO-RTG043 international Working Group, in which CIRA is being involved in order to improve its experience in dealing with aerothermodynamic databases at high altitude. In fact, in this framework, CIRA had the opportunity to compare its own results with the ones obtained by other partners of the group [1].

For both FTB-X and CEV configurations, the analyses were focused not only on the aerodynamic coefficients, but also on the heat loads, which can be critical already at high altitudes.

The study of the transitional flow regime presents theoretical and numerical difficulties. It is well known that the Navier-Stokes equations fail in rarefied regimes and a molecular approach such as the Direct Simulation Monte Carlo method (DSMC) is necessary. Moreover, slip flow boundary conditions have been implemented in CIRA CFD code H3NS in order to extend the validity of the continuum approach to the transitional flow regime, and bridgingformula models for the high altitude aerodynamics of a winged bodies have been developed. 


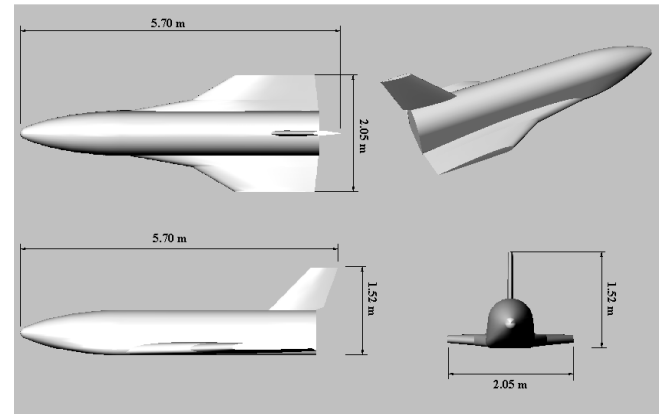

FIGURE 1. Views and dimensions FTB-X 3.9.2 FW50

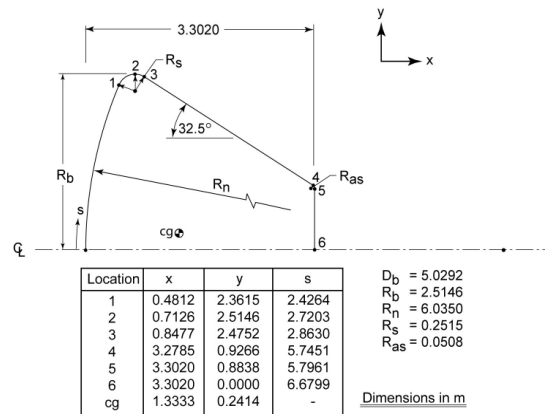

FIGURE 2. ORION Crew Exploration Vehicle

In order to validate the proposed approach, computations were performed also on the hollow cylinder flare test case of ONERA for which experiments and numerical results are fully available $[6,7,8]$, and on a test article that was used at CIRA for experimental tests in "Scirocco" Plasma Wind Tunnel (PWT) [9].

\section{COMPUTATIONAL METHODS}

\section{Computational Fluid Dynamic Method}

The continuum regime results have been obtained by using the CIRA Computational Fluid Dynamics (CFD) code H3NS developed at the Aerothermodynamics and Space Propulsion Laboratory [10]. The code solves full Reynolds Averaged Navier-Stokes (RANS) equations and considers the air flow in thermo-chemical nonequilibrium. Thermo-chemical and transport properties models are at the state of the art, and are fully described and referenced in [10]. The H3NS code is based on a finite volume approach with a cell centered formulation. The inviscid fluxes are computed by Flux Difference Splitting scheme and second order approximation is obtained with an Essentially Non Oscillatory (ENO) reconstruction of interface values. Time evolution is performed by an explicit multistage Runge-Kutta algorithm coupled with an implicit evaluation of the source terms.

To take into account the effects of rarefaction, the slip boundary conditions formulation proposed by Kogan [11] has been selected and implemented.

\section{Direct Simulation Monte Carlo Method}

The Direct Simulation Monte Carlo (DSMC) method used in this paper is based on the DS2V code by Bird [12].

The DSMC method considers the gas as made up of discrete particles represented by millions of simulated molecules, and it relies on the kinetic theory of gases. Movement and evolution of each molecule in the simulated physical space is the result of collisions with other molecules and with the body under study, in both cases exchanging momentum and energy. Excitation of rotational and vibrational degrees of freedom and chemical reactions can be also taken into account. The computational domain, including the test body, is divided in cells, which are used only for sampling the macroscopic properties and for selecting the colliding molecules. The movement of each molecule from a cell to another one is the product of the velocity (that is the resultant of the convective and thermal velocities) and a time step. Macroscopic thermo-fluid-dynamic quantities of the flow field (density, temperature, pressure and so on) are computed in each cell as an average over the molecules.

DS2V uses transient subcelles in which a transient background grid is built on a single cell and the collision routine, based on nearest-neighbor collisions, is applied. The resolution of the transient grid depends on the number of simulated molecules and, approximately, one simulated molecule corresponds to one subcell. The ratio of the local mean separation between collision partners to the local mean free path $(\mathrm{mcs} / \lambda)$ is provided by DS2V and it should be less than unity through the computational domain. Bird [12] suggests the value of 0.2 as a limit value.

\section{Bridging Formulas}

In this paragraph a brief description of Potter's formulas is reported [13]. The Potter's formulas, evaluating the aerodynamic coefficients $\mathrm{C}_{\mathrm{L}}$ and $\mathrm{C}_{\mathrm{D}}$ in the transitional regime, read: 


$$
\bar{C}_{L, D}=\left[2.6 /\left(2.6+P_{N L, D}^{1.6}\right)\right]^{0.5}
$$

where $\overline{\mathrm{C}}_{\mathrm{L}}$ and $\overline{\mathrm{C}}_{\mathrm{D}}$ are the normalized form of the drag and lift coefficients:

$$
\overline{\mathrm{C}}_{\mathrm{D}}=\frac{\left(\mathrm{C}_{\mathrm{D}}-\mathrm{C}_{\mathrm{Di}}\right)}{\left(\mathrm{C}_{\mathrm{Dfm}}-\mathrm{C}_{\mathrm{Di}}\right)}, \quad \overline{\mathrm{C}}_{\mathrm{L}}=\frac{\left(\mathrm{C}_{\mathrm{L}}-\mathrm{C}_{\mathrm{Li}}\right)}{\left(\mathrm{C}_{\mathrm{Lfm}}-\mathrm{C}_{\mathrm{Li}}\right)}
$$

$\mathrm{P}_{\mathrm{NL}}$ (for $\mathrm{C}_{\mathrm{L}}$ ) and $\mathrm{P}_{\mathrm{ND}}$ (for $\mathrm{C}_{\mathrm{D}}$ ) are parameters depending upon the vehicle geometry and flow conditions (i.e. free stream velocity, total enthalpy, etc.) and $\mathrm{C}_{\mathrm{Dfm}}, \mathrm{C}_{\mathrm{Lfm}}, \mathrm{C}_{\mathrm{Di}}$ and $\mathrm{C}_{\mathrm{Li}}$ are the drag and lift coefficients in continuum and free molecular regimes, respectively (see reference [13] for details)

Potter obtained these formulas by interpolating Space Shuttle re-entry flight data. For the purpose of the present paper both aerodynamic data and geometrical parameters referring to FTB-X have been used.

\section{ANALYSIS OF THE RESULTS}

\section{Shock Wave Boundary Layer Interactions}

The validation of the numerical methodologies has been conducted on prediction of local effects of rarefaction in hypersonic regime, and a classic experimental test case (the hollow cylinder flare) devoted to the study of shock wave boundary layer interaction has been firstly analyzed.

The hollow cylinder flare (FIGURE 3) is characterized by a sharp leading edge followed by a compression flare inclined by $30 \mathrm{deg}$ with respect to the cylinder, and a hollow cylindrical section. The model total length is $0.17 \mathrm{~m}$ and the reference length (L) is the distance between the leading edge and the beginning of the compression flare. The experiment was carried out in the R5Ch blow-down hypersonic wind tunnel at ONERA Chalais-Meudon [6], a massive CFD investigation has been performed by Marini [7] while a comparison between continuum and kinetic approach with respect to experimental results can be found in Markelov et al. [8].

Test conditions are reported in TABLE 1, and the surface temperature of the model is assumed to be constant and equal to $\mathrm{Tw}=293 \mathrm{~K}$. It is worth to underline that most of the flow domain is in continuum regime, except for the sharp leading edge and the regions within the boundary layer, where the Knudsen number is locally higher, being the reference length the boundary layer thickness, and therefore rarefaction effects are significant. FIGURE 3 shows the computed Mach number contours and streamlines obtained by using CFD with slip conditions: the strong viscous interaction at the cylinder leading edge is clearly predicted, as well as the shock wave boundary layer interaction

\begin{tabular}{|c|c|}
\hline \multicolumn{2}{|c|}{$\begin{array}{c}\text { TABLE } 1 \text { Hollow Cylinder Flare } \\
\text { Test Conditions }\end{array}$} \\
\hline $\mathrm{P}_{0}$ & $2.5 \times 10^{5} \mathrm{~Pa}$ \\
\hline $\mathrm{T}_{0}$ & $1050 \mathrm{~K}$ \\
\hline$M_{\infty}$ & 9.91 \\
\hline $\operatorname{Re}_{\infty} / m$ & $1.86 \times 10^{5}$ \\
\hline$K n_{\infty}^{\infty}$ & $9.35 \times 10^{-4} \mathrm{~m}$ \\
\hline$\lambda_{\infty}$ & $9.5 \times 10^{-4} \mathrm{~m}$ \\
\hline
\end{tabular}
around the corner with the related recirculation bubble.

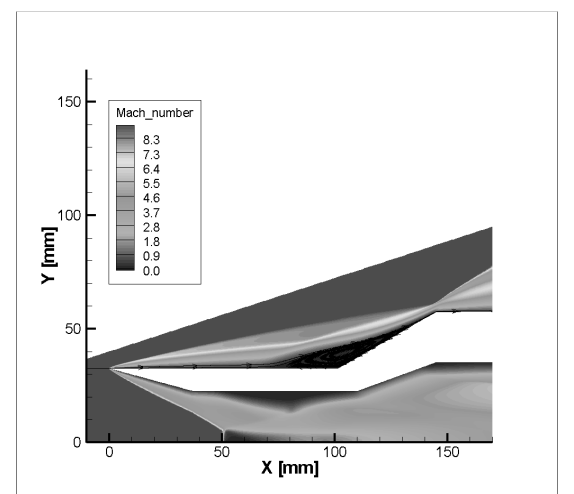

FIGURE 3. CFD Slip: Mach number contours and streamlines

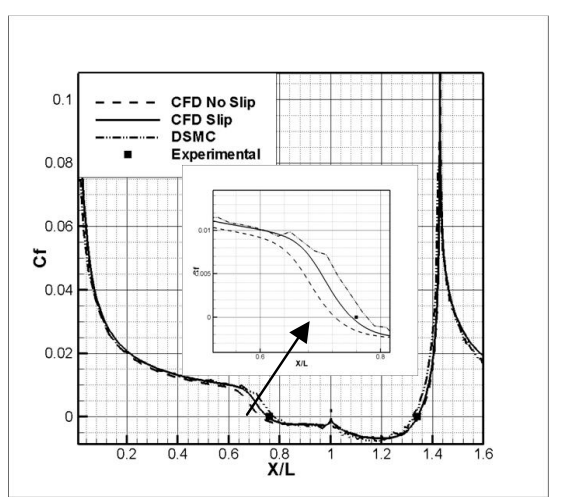

FIGURE 4. Skin friction coefficient distribution

From the analysis of the skin friction distributions, shown in FIGURE 4, it can be clearly seen that the three methodologies predict different separation lengths. In particular, the separation length is larger as the "amount of rarefaction of the method" decreases. In fact, the value of the non-dimensional abscissa of the separation point $\left(\mathrm{X}_{\mathrm{sep}} / \mathrm{L}\right)$ for CFD No Slip, CFD Slip and DSMC is, respectively, 0.7245, 0.7555 and 0.7748 . The experimental value 
(0.76) is comprised between CFD Slip and DSMC values as a proof of the local effects of rarefaction, being the local Knudsen number equal to $2 \cdot 17 \cdot 10^{-2}$.

Note that the reattachment point is not significantly affected by the computation methodology (see FIGURE 4).

A comparison of the results obtained by means of numerical simulations with the measured normalized density profiles inside the boundary layer is reported in FIGURE 5. In the cross section $\mathrm{X} / \mathrm{L}=0.3$ the agreement between numerical and experimental data in the case of Navier-Stokes with slip flow boundary conditions and DSMC is good, while it is fair for CFD with the classic boundary condition (CFD No Slip).

In particular, the shock wave angle predicted by classic CFD No Slip is higher with respect to the measured one.

Once the methodology has been checked on this reference test

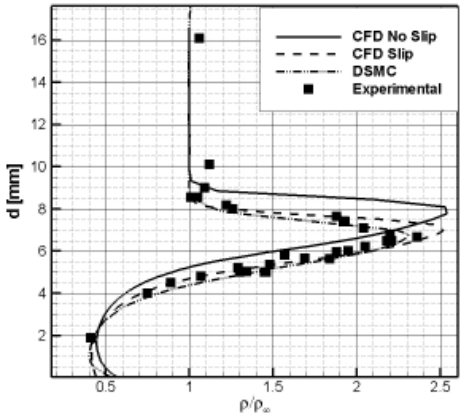

FIGURE 5. Density profile: $\mathrm{X} / \mathrm{L}=0.3$ case, a question arose about the possible rarefaction effects that can occur on CIRA Plasma Wind Tunnel "Scirocco", in particular for the test conditions characterized by the lower stagnation pressure and a unit freestream Knudsen number equal $1.47 \cdot 10^{-3}$.

For the present analysis, two dimensional computations have been carried out over the 20 deg full-scale flap of the EXPERT capsule (FIGURE 6) that will be tested in PWT facility (see [9] for details); the conditions $\mathrm{H}_{0}=35 \mathrm{MJ} / \mathrm{kg}, \mathrm{P}_{0}=2$ bar, corresponding to the lower freestream Knudsen number $\left(1.47 \cdot 10^{-3}\right)$, will be analyzed by comparing the results obtained with a classical Navier-Stokes approach with those of the DSMC method, in order to check possible effects of rarefaction on the separation length. The test chamber conditions, computed with the Navier-Stokes code and considering the largest conical nozzle $(1.95 \mathrm{~m}$ exit diameter), are $M_{\infty}=12.94, \mathrm{Re}_{\infty} / \mathrm{m}=9.03 \cdot 10^{3}$ and $\mathrm{T}_{\infty}=240 \mathrm{~K}$ while the wall has been considered at a fixed temperature of $300 \mathrm{~K}$.

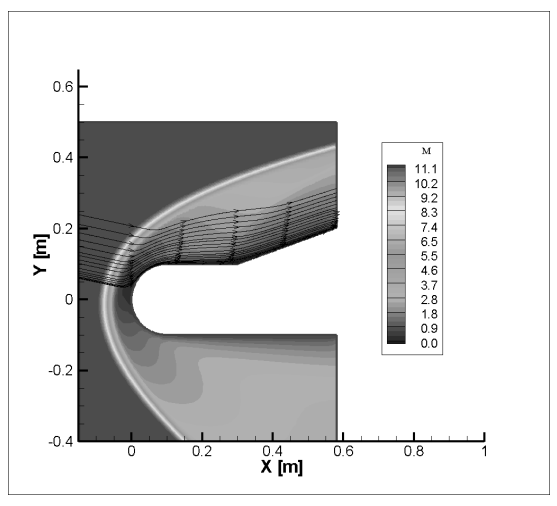

FIGURE 6. CFD: Mach number contours and streamlines

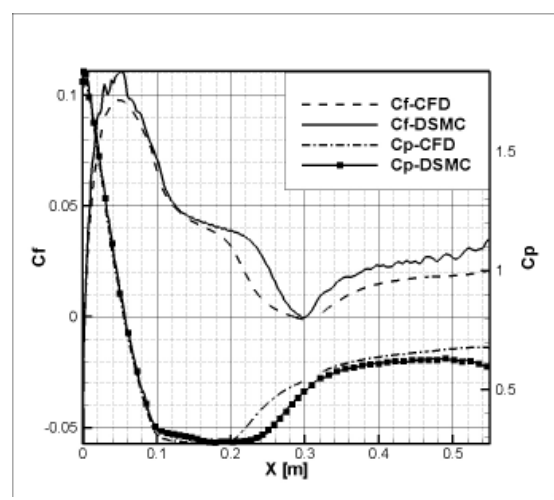

FIGURE 7. Skin friction and pressure coefficient distributions

By carefully examining FIGURE 6, and remembering the analysis performed for the hollow cylinder flare test case, the same considerations apply to the present case in high enthalpy conditions. In particular, a reduction of the separation length is observed with DSMC calculation as well as a slight reduction of the mechanical load acting on the flap (see FIGURE 7). As a conclusion, it must be stressed the fact that local rarefaction effects must be taken into account when designing plasma wind tunnel tests in "Scirocco" PWT at limit conditions of the facility envelope, in particular for very low pressures and high enthalpies.

\section{Effects of Rarefaction on a Winged Hypersonic Re-entry Vehicle}

As already discussed, an important aspect to be taken into account in the design of a re-entry vehicle is the evaluation of the effects of rarefaction on the aerodynamic coefficients. At high altitudes, in transitional regime, it is well known that a decrease of the lift and an increase of the drag occur. The correct evaluation of lift and drag is particularly important in the design of the trajectory and of the Reaction Control System of a winged re-entry vehicle like FTB-X, that is characterized by a high aerodynamic efficiency. 


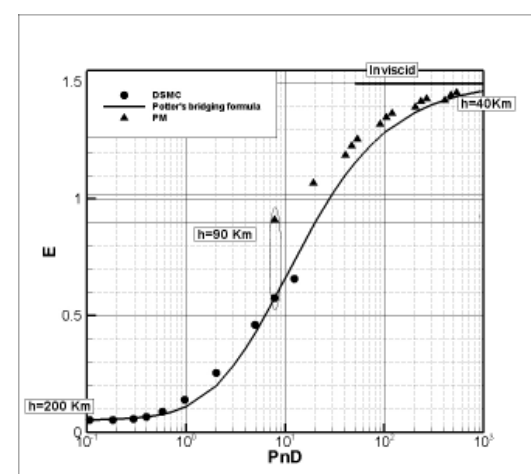

FIGURE 8. Aerodynamic efficiency of FTB$\mathrm{X}(1.1 .2)$ as a function of PND: $\alpha=30 \mathrm{deg}$
During the trade-off phase of the FTB-X vehicle several configurations were rapidly analyzed, mainly using engineering methods as Panel Method (PM) in continuum regime and Potter bridging formulas in transitional regime. The results obtained on the preliminary configuration 1.1 .2 have been compared with some DSMC and CFD simulations, in order to verify the reliability of engineering methods. In FIGURE 8 the results of this comparison are shown for $\alpha=30 \mathrm{deg}$, that corresponds to the maximum efficiency in rarefied conditions. The aerodynamic efficiency $\mathrm{E}=\mathrm{C}_{\mathrm{L}} / \mathrm{C}_{\mathrm{D}}$ computed by DSMC, PM and the Potter bridging formula is plotted as function of the $\mathrm{P}_{\mathrm{ND}}$ parameter. The inviscid limit computed by CFD is also shown and a free molecular calculation has been performed, since in order to apply Potter's formula the inviscid limit and the free molecular one must be known.

FIGURE 8 shows that, for high values of $\mathrm{P}_{\mathrm{ND}}$ (i.e. at low altitude=h), the continuum results $(\mathrm{PM})$ are in good agreement with those from the bridging formula. However, when altitude increases and $\mathrm{P}_{\mathrm{ND}}$ decreases, the PM results tend to significantly overestimate the efficiency. For example, when $\mathrm{P}_{\mathrm{ND}}=7.8$ (corresponding to $\mathrm{h}=90 \mathrm{~km}$ ) the value of E from PM is nearly 0.91, while the one from DSMC is about 0.58 , then PM overestimating E of about $60 \%$. It can be concluded that, for $\mathrm{P}_{\mathrm{ND}}$ approximately lower than 40 (i.e. $\mathrm{h}>60 \mathrm{~km}$ ), it is important to take into account for the FTB-X trajectory the rarefaction effects. This can be done by using a DSMC code or alternatively the bridging formulas, that allow having a more rapid and sufficiently reliable estimation of the aerodynamic coefficients; the results can be successively verified by means of DSMC computations only in selected conditions.

\section{Effects of Rarefaction on ORION CEV Capsule Heating}

An aerothermal analysis of ORION CEV capsule has been conducted in transitional regime in order to verify the predicting capabilities of aerothermal loads acting on a blunt re-entry vehicle, in particular in the range of altitudes where both CFD and DSMC approaches could be used.

A point of ORION re-entry trajectory in transitional regime $\left(\mathrm{h}=85 \mathrm{~km}, \mathrm{Kn}_{\infty}=0.0019\right)$ has been selected as reference case to compare the results obtained by means of the continuum approach and those obtained by means of the DSMC code with the following conditions: freestream velocity of $7.6 \mathrm{~km} / \mathrm{s}$ and fixed $\mathrm{T}_{\mathrm{w}}=1184 \mathrm{~K}$.

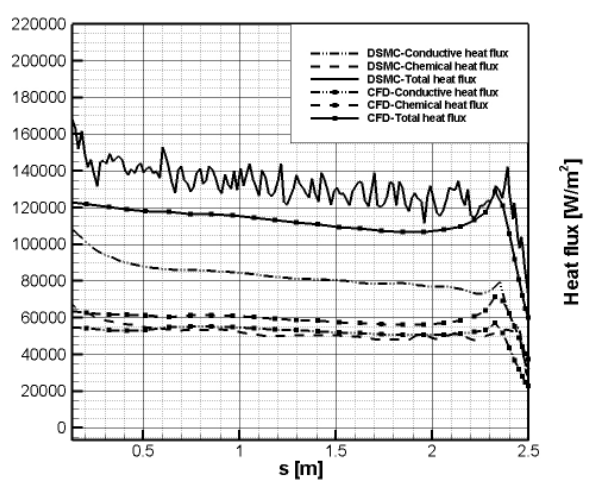

FIGURE 9. Heat flux; $\mathrm{h}=85 \mathrm{~km}$; fully catalytic wall

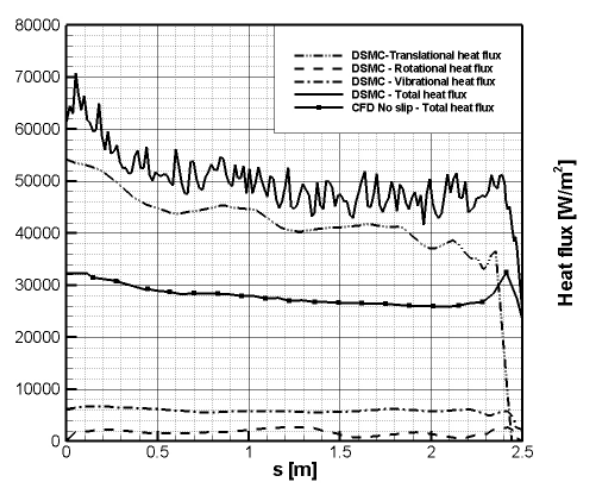

FIGURE 10. Heat flux; $h=85 \mathrm{~km}$; non-catalytic wall

An evaluation of the single contributions to the global heat flux (fully catalytic wall case) is shown FIGURE 9. In particular, it can be observed that the chemical contribution to the heat flux calculated by CFD is very close to DSMC one, while the conductive one is lower. This difference on global heat flux is confirmed from non-catalytic wall case showed in FIGURE 10. It is interesting to observe that in the non catalytic case the percentage difference between CFD and DSMC results increases from about $10 \%$ to $60 \%$, but the absolute value of this difference is the same as for fully catalytic wall; this confirms that the discrepancy is not due by the chemical part of the heat flux, but from the conductive one. 


\section{CONCLUSIONS}

The purpose of the present research activity was the study of the predicting capabilities for the evaluation of aero-thermal loads acting on a space re-entry vehicle at high altitude. The results obtained by using a continuum approach were compared with those given by a DSMC code. In particular, two configurations have been analyzed: the CIRA winged USV FTB-X experimental re-entry vehicle and the NASA ORION CEV capsule.

A preliminary validation of the different methods has been conducted by evaluating the effects of rarefaction on a classical test case, the hollow cylinder flare in ONERA R5Ch wind tunnel conditions. The CFD code with slip flow boundary conditions has shown good predicting capabilities of the size of the recirculation bubble and density profiles. Definitively, this test case, simulated with three different methodologies (CFD no slip, CFD with slip flow boundary conditions and DSMC), has shown that CFD with slip flow boundary conditions can be a good compromise between computational cost and accuracy. The same considerations apply to a test case, devoted to the study of the shock wave boundary layer interaction, planned in the CIRA Plasma Wind Tunnel "Scirocco".

After this preliminary analysis, the aerodynamic behaviour of the winged experimental re-entry vehicle FTB-X has been evaluated in the altitude range $90-110 \mathrm{~km}$, in transitional regime. Computations have been performed by means of a 3D DSMC code and a panel method, allowing a fast prediction of the aerodynamic coefficients. In addition, the Potter's bridging formulas have been used to correlate continuum and free molecular results. The panel methods resulted to be lacking at altitudes higher than $60 \mathrm{~km}$ for the FTB-X re-entry trajectory; the Potter's bridging formula provided instead satisfactory results compared with DSMC data. Finally, the aerothermodynamics in rarefied conditions has been analyzed for the ORION CEV reentry capsule, in the transitional part of the re-entry trajectory. The analysis has been conducted at the altitude of $85 \mathrm{~km}$. The surface heat flux calculated by DSMC code is about $10 \%$ higher than CFD one for the fully catalytic wall assumption, while this difference raises to about $60 \%$ in the case of non catalytic wall, being the discrepancy mainly due to the conductive heat flux.

Definitively, the experience done with the present research activity allowed to develop the know-how needed to cover an entire re-entry trajectory, from the higher altitudes (free molecular flow regime), through the transitional zone, till the continuum flow. This skill will be important not only in the next phases of FTB-X design, but also in the aerodynamic analysis of any space vehicle re-entering the atmosphere.

\section{REFERENCES}

1. R. Votta, A. Schettino, G. Ranuzzi and S. Borrelli, "Hypersonic Low-Density Aerothermodynamics of Orion-Like Exploration Vehicle", The Journal of Spacecraft and Rockets, Vol. 46 No. 4, July-August 2009, pp. 781-787.

2. R. Votta, G. Ranuzzi, M. Di Clemente, A. Schettino and M. Marini, "Evaluation of Local Effects of Transitional Knudsen Number on Shock Wave Boundary Layer Interactions", 39th AIAA Thermophysics Conference, Miami (USA), AIAA-20074545, June 2007.

3. G. Zuppardi, F. Costagliola, A. Rega, A. Schettino, R. Votta, "Evaluation of Rarefaction Effects on a Winged, Hypersonic Re-entry Vehicle", Proceedings of the 14th AIAA/AHI, International Space Planes and Hypersonic Systems and Technologies Conference, Canberra (AU), AIAA-2006-8114, November 2006.

4. A. Schettino et al., "Mission Trade- Off Analysis of the Italian USV Re- Entry Flying Test Bed", Proceedings of the 14th AIAA/AHI International Space Planes and Hypersonic Systems and Technologies Conference, Canberra (AU), AIAA-20068017, November 2006.

5. Moss, J. N., Boyles, K. A., and Greene, F. A., "Blunt Body Aerodynamics for Hypersonic Low Density Flows, "Proceedings of 25th Symposium on Rarefied Gas Dynamics, edited by Ivanov M. S. and Rebrov A., K., Vol. 1, Siberian Branch of the Russian Academy of Sciences Saint-Petersburg (RU) 2006, pp. 753-758.

6. Chanetz., B., "ONERA hypersonic test-cases in the framework of the Working Group 18th Hypersonic experimental and computational capability", AGARD Advisory Report 319, Vol. 2, 1996-91.

7. Marini, M., "H09 Viscous Interaction at a Cylinder/Flare Junction”, Third FLOWNET Workshop, , Marseille, 2002.

8. Markelov, G., N., Kudryavtsev A. N., Ivanov, M., S., "Continuum and Kinetic Simulation of Laminar Separated Flow at Hypersonic Speeds", The Journal of Spacecraft and Rockets, Vol. 37 No. 4, July-August 2000.

9. Di Clemente M., Marini M., Schettino A., "Shock Wave Boundary Layer Interaction in EXPERT Flight Conditions and Scirocco PWT", $13^{\text {th }}$ AIAA/CIRA International Space Planes and Hypersonics Systems and Technologies Conference, Capua, Italy, 2005.

10. G. Ranuzzi and S. Borreca, "CLAE Project. H3NS: Code Development Verification and Validation”, CIRA-CF-06-1017, 2006.

11. N. M. Kogan, Rarefied Gas Dynamics, New York, Plenum 1969.

12. G. A. Bird, Molecular Gas Dynamics and the Direct Simulation of Gas Flows, Oxford: Clarendon 1994.

13. Potter, J. L., "Procedure for Estimating Aerodynamics of Three-Dimensional Bodies in Transitional Flow," Progress in Astronautics and Aeronautics, Vol. 118, 1988 pp 484-492. 